\title{
Index for subgroups of the group of units in number fields
}

\author{
by
}

\author{
Tsutomu Shimada (Yamato)
}

We define a sequence of rational integers $u_{i}(E)$ for each finite index subgroup $E$ of the group of units in some finite Galois number fields $K$ in which prime $p$ ramifies. For two subgroups $E^{\prime} \subset E$ of finite index in the group of units of $K$ we prove the formula $v_{p}\left(\left[E: E^{\prime}\right]\right)=\sum_{i=1}^{r}\left\{u_{i}\left(E^{\prime}\right)-u_{i}(E)\right\}$. This is a generalization of results of P. Dénes [3], [4] and F. Kurihara [5].

Introduction. Let $p$ be an odd prime number, $\mathbb{Q}$ and $\mathbb{Z}$ the field of rational numbers and the ring of rational integers, respectively. For each unit $\varepsilon$ of $\mathbb{Q}\left(\zeta_{p}\right)$ which is not in $\mathbb{Z}$, there exist rational integers $a, b$ and $c$ satisfying $\varepsilon \equiv a+b\left(1-\zeta_{p}\right)^{c} \bmod \left(1-\zeta_{p}\right)^{c+1}, a b \not \equiv 0 \bmod p$ and $c \not \equiv 0 \bmod (p-1)$, where $c$ is uniquely determined by $\varepsilon$. P. Dénes [2] defined the $p$-character of the Bernoulli numbers to be the rational integers $u_{2}, u_{4}, \ldots, u_{p-3}$ such that $B_{i p^{j}} \equiv 0 \bmod p^{2 j+1}$ for $0 \leq j<u_{i}$ and $B_{i p^{u_{i}}} \not \equiv 0 \bmod p^{2 u_{i}+1}$, where $i=2,4, \ldots, p-3$, and proved the following results under the assumption that the $p$-character of the Bernoulli numbers exists:

Theorem A. There exists a basis $\left\{\theta_{2}, \theta_{4}, \ldots, \theta_{p-3}\right\}$ for the group of units of $\mathbb{Q}\left(\zeta_{p}\right)^{+}$modulo $\{ \pm 1\}$ such that

$$
\theta_{i} \equiv a_{i}+b_{i}\left(1-\zeta_{p}\right)^{c_{i}} \bmod \left(1-\zeta_{p}\right)^{c_{i}+1}
$$

with $c_{i}=i+(p-1) u_{i}^{\prime}$ for some integer $0 \leq u_{i}^{\prime} \leq u_{i}$.

TheOrem B. We have

$$
v_{p}\left(h\left(\mathbb{Q}\left(\zeta_{p}\right)^{+}\right)\right)=\sum_{i=2, \text { even }}^{p-3}\left(u_{i}-u_{i}^{\prime}\right) .
$$

Here $h(*)$ denotes the class number of a field $*$ and $v_{p}$ the $p$-adic valuation normalized by $v_{p}(p)=1$.

1991 Mathematics Subject Classification: 11R21, 11R29. 
L. C. Washington [6] showed that $u_{i}=v_{p}\left(L_{p}\left(1, \omega^{i}\right)\right), i=2,4, \ldots, p-3$, and then proved Dénes' assumption stated above, where $\omega$ is the Teichmüller character. Furthermore, Washington gave simple proofs of the theorems above. In [5], F. Kurihara generalized the results above to a subfield $K_{n+1}$ of $\mathbb{Q}\left(\zeta_{p^{n+1}}\right)^{+}, 0 \leq n \in \mathbb{Z}$, and showed the following two theorems.

THEOREM C. Let $E$ be a subgroup of $E_{K_{n+1}}$, the group of units of $K_{n+1}$, such that $\left[E_{K_{n+1}}: E\right]$ is finite. Then there exists a basis $\left\{\theta_{1}, \ldots, \theta_{r}\right\}$ for $E$ modulo $\{ \pm 1\}$ such that

$$
\theta_{i}^{p^{n}} \equiv a_{i}+b_{i} \pi^{c_{i}} \bmod \pi^{c_{i}+1}, \quad i=1, \ldots, r,
$$

with $c_{i}=i+\frac{1}{2 d} \varphi\left(p^{n+1}\right) u_{i}$ for some rational integer $u_{i} \geq 0$.

Here $d=\left[\mathbb{Q}\left(\zeta_{p^{n+1}}\right)^{+}: K_{n+1}\right], r=\operatorname{rank} E_{K_{n+1}}=\frac{1}{2 d} \varphi\left(p^{n+1}\right)-1$ and $\pi$ is the image of $\left(1-\zeta_{p^{n+1}}\right)\left(1-\zeta_{p^{n+1}}^{-1}\right)$ by the norm from $\mathbb{Q}\left(\zeta_{p^{n+1}}\right)^{+}$to $K_{n+1}$. Now since $c_{i}$ and hence $u_{i}$ depends only on $E$, it is denoted by $c_{i}(E)$ and $u_{i}(E)$, respectively.

Theorem D. Let $E$ be a subgroup of $E_{K_{n+1}}$ and $E^{\prime}$ a subgroup of $E$. Suppose that the index $\left[E_{K_{n+1}}: E^{\prime}\right]$ is finite. Then

$$
v_{p}\left(\left[E: E^{\prime}\right]\right)=\sum_{i=1}^{r}\left\{u_{i}\left(E^{\prime}\right)-u_{i}(E)\right\} .
$$

Considering the case where $K_{n+1}=\mathbb{Q}\left(\zeta_{p^{n+1}}\right)^{+}, E=E_{K_{n+1}}$ and $E^{\prime}$ is the group of cyclotomic units in the sense of Sinnott, we see that Theorem D is a generalization of Theorem B.

Our aim is to prove similar results in some other number fields: the composite of two Galois extensions of finite degree over $\mathbb{Q}$, one unramified at $p$ and the other totally ramified.

Now we introduce some notations. Let $K_{T}$ be a finite Galois extension over $\mathbb{Q}$ which is unramified at $p$ and $K_{V}$ a finite Galois extension which is totally ramified at $p$. Let $K=K_{T} K_{V},\left[K_{T}: \mathbb{Q}\right]=d_{K}$ and $\left[K_{V}: \mathbb{Q}\right]=e_{K}$. We fix an embedding of $K$ into $\mathbb{C}$, the field of complex numbers. Let $J$ denote the restriction of the complex conjugation to $K$. Let $K^{+}$and $K_{T}^{+}$be the fixed field of $\langle J\rangle$ in $K$ and $K_{T}$, respectively. Let $\wp_{1}, \ldots, \wp_{g}$ be the primes of $K_{T}$ over $p, \wp_{i}^{\prime}(i=1, \ldots, g)$ the primes of $K$ over $\wp_{i}$, and $\wp$ the unique prime of $K_{V}$ over $p$. Then $\wp_{i}=\wp_{i}^{\prime e_{K}}$ and $(p)=\wp_{1} \ldots \wp_{g}=\left(\wp_{1}^{\prime} \ldots \wp_{g}^{\prime}\right)^{e_{K}}=\wp^{e_{K}}$ in the ring $O_{K}$ of integers of $K$. Let $E_{*}$ be the group of units of a field $*$. Let $\delta$ be the least natural number $a$ that satisfies $e_{K} /(p-1)<p^{a-1}$.

Throughout this paper, we assume the following three conditions:

$\left(A_{1}\right) \wp$ is a principal ideal.

$\left(\mathrm{A}_{2}\right)$ The exponent of the torsion part of $E_{K^{+}} / E_{K_{T}^{+}}$is prime to $p$.

$\left(\mathrm{A}_{3}\right)$ The Leopoldt conjecture is valid for $K$ and $p$. 
Then we may write $\wp=\left(\pi_{K}\right)$ with some $\pi_{K} \in O_{K_{V}}$. Let $m$ be the exponent in $\left(\mathrm{A}_{2}\right)$.

Our main result is the following:

Theorem E. Let $E$ be a subgroup of $E_{K^{+}}$such that $E \supset E_{K_{T}^{+}}$and $\left[E_{K^{+}}: E\right]<\infty$. Then there exists a basis $\left\{\eta_{1}, \ldots, \eta_{r}\right\}$ of $E^{m p^{\delta} e_{K}} E_{K_{T}^{+}}$modulo $E_{K_{T}^{+}}$such that

$$
\eta_{i} \equiv a_{i}+b_{i} \pi_{K}^{c_{i}} \bmod \pi_{K}^{c_{i}+1}, \quad i=1, \ldots, r=\operatorname{rank} E_{K^{+}} / E_{K_{T}^{+}},
$$

where $a_{i}$ and $b_{i}$ belong to the ring $O_{K_{T}}$ of integers of $K_{T}, a_{i} \not \equiv 0$ modulo any prime over $p, b_{i} \not \equiv 0 \bmod p$ and $c_{i}$ is a natural number such that $c_{i} \not \equiv$ $0 \bmod e_{K}$.

Further, let $u_{i}(E)=\left[\frac{c_{i}}{e_{K}}\right]$ (Gauss symbol) and $E^{\prime}$ be a subgroup of $E$ such that $E^{\prime} \supset E_{K_{T}^{+}}$and $\left[E_{K^{+}}: E^{\prime}\right]<\infty$. Then

$$
v_{p}\left(\left[E: E^{\prime}\right]\right)=\sum_{i=1}^{r}\left\{u_{i}\left(E^{\prime}\right)-u_{i}(E)\right\} .
$$

REMARK 1. Let $\eta$ be any element of $E_{K^{+}} \backslash K_{T}$ such that $\eta^{a} \in E_{K_{T}^{+}}$ for some natural number $a$. Then, for any $\sigma \in \operatorname{Gal}\left(K / K_{T}\right),\left(\eta^{\sigma} / \eta\right)^{a}=$ $\left(\eta^{a}\right)^{\sigma} / \eta^{a}=\eta^{a} / \eta^{a}=1$. So, $\eta^{\sigma-1}$ is an ath root of unity. Moreover, when $K$ is a real or $C M$-field, $\eta^{\sigma-1}$ is real, hence \pm 1 and $\left(\eta^{2}\right)^{\sigma-1}=1$. Thus, $\eta^{2} \in E_{K_{T}^{+}}$and $m$ equals 1 or 2 , hence in this case it is prime to $p$, i.e. $\left(\mathrm{A}_{2}\right)$ is valid.

REMARK 2. When $K=\mathbb{Q}\left(\zeta_{a p^{n}}\right)$ with $a$ prime to $p$, then $K_{T}=\mathbb{Q}\left(\zeta_{a}\right)$. Note that the condition $\left(\mathrm{A}_{3}\right)$ is valid by the theorem of $\mathrm{A}$. Brumer [1]. Let $E=E_{K^{+}}$and $E^{\prime}=C_{K}^{+} E_{K_{T}^{+}}$, where $C_{K}$ is the group of cyclotomic units and $C_{K}^{+}=C_{K} \cap K^{+}$. Then, since $v_{p}\left(\left[E: E^{\prime}\right]\right)=v_{p}\left(h\left(K^{+}\right) / h\left(K_{T}^{+}\right)\right)$, we get a generalization of Theorem $\mathrm{B}$ :

$$
v_{p}\left(h\left(\mathbb{Q}\left(\zeta_{a p^{n}}\right)^{+}\right) / h\left(\mathbb{Q}\left(\zeta_{a}\right)^{+}\right)\right)=\sum_{i=1}^{r}\left\{u_{i}\left(E^{\prime}\right)-u_{i}(E)\right\} .
$$

1. The Fermat quotient and the level of unit. Let the notations be as in the introduction. Note that $K_{V}=\mathbb{Q}\left(\pi_{K}\right)$. Let $f(X)=X^{e_{K}}+$ $c_{e_{K}-1}^{\prime} X^{e_{K}-1}+\ldots+c_{1}^{\prime} X+c_{0}^{\prime} \in \mathbb{Z}[X]$ be the minimal polynomial of $\pi_{K}$ which is of Eisenstein type. That is, $c_{e_{K}-1}^{\prime} \equiv \ldots \equiv c_{0}^{\prime} \equiv 0 \bmod p$ and $c_{0}^{\prime} \not \equiv 0 \bmod p^{2}$. We write $c_{i}^{\prime}=-p c_{i}\left(i=0,1, \ldots, e_{K}-1\right)$. Then

$$
\pi_{K}^{e_{K}} \equiv p c_{0} \bmod \pi_{K}^{e_{K}+1} \quad \text { and } \quad p \equiv b_{0} \pi_{K}^{e_{K}} \bmod \pi_{K}^{e_{K}+1} \text {, }
$$

where $b_{0}$ is the natural number such that $c_{0} b_{0} \equiv 1 \bmod p$ and $1 \leq b_{0} \leq p-1$. 
For any $z \in O_{K} \backslash K_{T}$ which is prime to $p$, we define

$c(z)=\max \left\{0 \leq c \in \mathbb{Z}: z \equiv x+y \pi_{K}^{c} \bmod \pi_{K}^{c+1}\right.$ with some $\left.x, y \in O_{K_{T}}\right\}$.

It can be easily seen that $1 \leq c(z)<\infty$. Furthermore, we define $c(z)=\infty$ for $z \in O_{K_{T}}$.

When $x_{0}$ and $y_{0}$ give $c(z)\left(z \in O_{K} \backslash K_{T}\right)$, it is clear that

$$
x_{0} \not \equiv 0 \bmod \wp_{i} \quad(i=1, \ldots, g) \quad \text { and } \quad y_{0} \not \equiv 0 \bmod p .
$$

If $c(z)$ is a multiple of $e_{K}$, then writing $c(z)=c e_{K}$ with a natural number $c$, we have

$$
z \equiv x_{0}+y_{0} \pi_{K}^{c e_{K}} \equiv x_{0}+y_{0} p^{c} c_{0}^{c} \bmod \pi_{K}^{c e_{K}+1},
$$

which contradicts the maximality of $c(z)$. Thus, we get $c(z) \not \equiv 0 \bmod e_{K}$.

To sum up, we have the following:

Lemma 1.1. For any $z \in O_{K} \backslash K_{T}$ which is prime to $p$,

$$
c(z)=\max \left\{0 \leq c \in \mathbb{Z}: z \equiv x+y \pi_{K}^{c} \bmod \pi_{K}^{c+1} \text { for some } x, y \in O_{K_{T}}\right\}
$$

is a natural number which depends only on $z$ (it does not depend on the choice of $\left.\pi_{K}\right)$ and $c(z) \not \equiv 0 \bmod e_{K}$. Let $x_{0}$ and $y_{0}$ be elements in $O_{K_{T}}$ giving $c(z)$. Then $x_{0} \not \equiv 0 \bmod \wp_{i}(i=1, \ldots, g)$ and $y_{0} \not \equiv 0 \bmod p$, and further, $x_{0}$ and $y_{0}$ are uniquely determined by $z$ modulo $\pi_{K}^{c(z)+1}$ and $p$, respectively.

Let $\eta \in E_{K} \backslash K_{T}$. Let $\eta \equiv x+y \pi_{K}^{c(\eta)} \bmod \pi_{K}^{c(\eta)+1}$ be a congruence giving $c(\eta)$ according to Lemma 1.1. Then, in the following, we call $c(\eta)$ the level of $\eta$ and $(y / x) \bmod p \in O_{K_{T}} /(p)$ the (generalized) Fermat quotient of $\eta$ and we denote the latter by $f(\eta)$. Of course they are uniquely determined by $\eta$.

In the rest of this section, we present several elementary properties of the level and the Fermat quotient.

The next lemma immediately follows from the definitions of the Fermat quotient and the level.

Lemma 1.2. (1) For any $\eta \in E_{K} \backslash K_{T}$ and any natural number a prime to $p$, we have $c\left(\eta^{J}\right)=c(\eta), c\left(\eta^{a}\right)=c(\eta), f\left(\eta^{a}\right)=a f(\eta)$ and $c\left(\eta^{-1}\right)=c(\eta)$, $f\left(\eta^{-1}\right)=-f(\eta)$.

(2) Let $\eta_{1}$ and $\eta_{2}$ be elements in $E_{K} \backslash K_{T}$ such that $c\left(\eta_{1}\right)<c\left(\eta_{2}\right)$. Then

$$
c\left(\eta_{1} \eta_{2}\right)=c\left(\eta_{1}\right) \quad \text { and } \quad f\left(\eta_{1} \eta_{2}\right)=f\left(\eta_{1}\right) .
$$

(3) Let $\eta_{1}, \ldots, \eta_{s}$ be elements in $E_{K} \backslash K_{T}$ such that $c\left(\eta_{1}\right)=\ldots=c\left(\eta_{s}\right)$ and $f\left(\eta_{1}\right)+\ldots+f\left(\eta_{s}\right) \not \equiv 0 \bmod p$. Then

$$
c\left(\eta_{1} \ldots \eta_{s}\right)=c\left(\eta_{1}\right) \quad \text { and } \quad f\left(\eta_{1} \ldots \eta_{s}\right)=f\left(\eta_{1}\right)+\ldots+f\left(\eta_{s}\right) .
$$

Lemma 1.3. If $K_{V}$ is imaginary, then $\pi_{K}^{J} \equiv-\pi_{K} \bmod \pi_{K}^{2}$. 
Proof. By the definition of $\pi_{K}$, we have $\left(\pi_{K}^{J}\right)=\left(\pi_{K}\right)$. So, there exists $u \in E_{K_{V}}$ such that $\pi_{K}^{J}=\pi_{K} u$. We have $\pi_{K}=\left(\pi_{K}^{J}\right)^{J}=\left(\pi_{K} u\right)^{J}=\pi_{K} u u^{J}$. Thus, $u u^{J}=1$.

First, we assume $u \notin K_{T}$. Let $u \equiv a+b \pi_{K}^{c(u)} \bmod \pi_{K}^{c(u)+1}$ according to Lemma 1.1. For any $\sigma \in \operatorname{Gal}\left(K / K_{V}\right), u^{\sigma}=u, \pi_{K}^{\sigma}=\pi_{K}$ and $u \equiv$ $a^{\sigma}+b^{\sigma} \pi_{K}^{c(u)} \bmod \pi_{K}^{c(u)+1}$. Because $O_{K_{V}} /\left(\pi_{K}\right)=\mathbb{Z} /(p)$, we can always write $u \equiv a+b \pi_{K}^{c} \bmod \pi_{K}^{c+1}$ where $a, b \in \mathbb{Z}$ are prime to $p$. Then $1=u u^{J} \equiv$ $a^{2} \bmod \pi_{K}$, so $a \equiv \pm 1 \bmod p$. Since $\pi_{K}^{J} \equiv a \pi_{K} \bmod \pi_{K}^{2}$, we have $u^{J} \equiv a+$ $b a^{c(u)} \pi_{K}^{c(u)} \bmod \pi_{K}^{c(u)+1}$. By Lemma 1.2, $f\left(u^{-1}\right)=f\left(u^{J}\right) \equiv b a^{c(u)-1} \bmod p$ and $f\left(u^{-1}\right)=-f(u) \equiv-b a^{-1} \bmod p$. This means $a^{c(u)} \equiv-1 \bmod p$, so that $a \equiv-1 \bmod p$ and the lemma is proved in this case.

Secondly, we assume $u \in K_{T}$. Then $u \in K_{T} \cap K_{V}=\mathbb{Q}$ and $u= \pm 1$. Now, $\pi_{K}^{J} \neq \pi_{K}$ by our assumption, so that $u=-1$. The proof is complete.

Lemma 1.4. For any $\eta \in E_{K} \backslash K_{T}$ we have $c\left(\eta^{p^{\delta}}\right)>e_{K} /(p-1)$. If $c(\eta)>e_{K} /(p-1)$, then $c\left(\eta^{p^{a}}\right)=c(\eta)+a e_{K}$ and $f\left(\eta^{p^{a}}\right)=b_{0}^{a} f(\eta)$ for all natural numbers $a$. Here $\delta$ and $b_{0}$ are as in the introduction.

Proof. Let $\eta \equiv x+y \pi_{K}^{c(\eta)} \bmod \pi_{K}^{c(\eta)+1}$ according to Lemma 1.1. Then there exists $y_{1} \in O_{K}$ such that $\eta=x+y_{1} \pi_{K}^{c(\eta)}$ and $y_{1} \equiv y \bmod \pi_{K}$. So,

$$
\begin{aligned}
\eta^{p}= & x^{p}+p x^{p-1} y_{1} \pi_{K}^{c(\eta)} \\
& +\left(\begin{array}{c}
p \\
2
\end{array}\right) x^{p-2} y_{1}^{2} \pi_{K}^{2 c(\eta)}+\ldots+\left(\begin{array}{c}
p \\
p-1
\end{array}\right) x y_{1}^{p-1} \pi_{K}^{(p-1) c(\eta)}+y_{1}^{p} \pi_{K}^{p c(\eta)} .
\end{aligned}
$$

Since the $\pi_{K}$-orders of terms on the right hand side are

$$
0, e_{K}+c(\eta), e_{K}+2 c(\eta), \ldots, e_{K}+(p-1) c(\eta) \text { and } p c(\eta),
$$

it follows that

$$
c\left(\eta^{p}\right) \geq \min \left\{e_{K}+c(\eta), p c(\eta)\right\} \geq \min \left\{\frac{e_{K}}{p-1}, p c(\eta)\right\} .
$$

Further,

$$
\begin{aligned}
c\left(\eta^{p^{2}}\right) & \geq \min \left\{\frac{e_{K}}{p-1}, p c\left(\eta^{p}\right)\right\} \\
& \geq \min \left\{\frac{e_{K}}{p-1}, p \min \left\{\frac{e_{K}}{p-1}, p c(\eta)\right\}\right\}=\min \left\{\frac{e_{K}}{p-1}, p^{2} c(\eta)\right\} .
\end{aligned}
$$

For all natural numbers $a$, we get by induction

$$
c\left(\eta^{p^{a}}\right) \geq \min \left\{\frac{e_{K}}{p-1}, p^{a} c(\eta)\right\} .
$$


Since $c\left(\eta^{p^{\delta-1}}\right) \geq e_{K} /(p-1),(2)$ means that

$$
c\left(\eta^{p^{\delta}}\right) \geq \min \left\{e_{K}+c\left(\eta^{p^{\delta-1}}\right), p c\left(\eta^{p^{\delta-1}}\right)\right\}>\frac{e_{K}}{p-1} .
$$

When $e_{K} /(p-1)<c(\eta)$, we have

$$
e_{K}+c(\eta)<p c(\eta) \text { and } \eta^{p} \equiv x^{p}+p x^{p-1} y_{1} \pi_{K}^{c(\eta)} \bmod \pi_{K}^{e_{K}+c(\eta)+1} .
$$

Now from (1), we have

$$
\eta^{p} \equiv x^{p}+b_{0} x^{p-1} y \pi_{K}^{e_{K}+c(\eta)} \bmod \pi_{K}^{e_{K}+c(\eta)+1} .
$$

So, we conclude that $c\left(\eta^{p}\right) \geq c(\eta)+e_{K}$.

Suppose $c\left(\eta^{p}\right)>c(\eta)+e_{K}$. Let $\eta^{p} \equiv x_{2}+y_{2} \pi_{K}^{c\left(\eta^{p}\right)} \bmod \pi_{K}^{c\left(\eta^{p}\right)+1}$ according to Lemma 1.1. Then

$$
x_{2} \equiv \eta^{p} \equiv x^{p}+b_{0} x^{p-1} y \pi_{K}^{e_{K}+c(\eta)} \bmod \pi_{K}^{e_{K}+c(\eta)+1}
$$

and

$$
x_{2}-x^{p} \equiv b_{0} x^{p-1} y \pi_{K}^{e_{K}+c(\eta)} \bmod \pi_{K}^{e_{K}+c(\eta)+1} .
$$

Take a prime $\wp_{i}^{\prime}$ dividing $\pi_{K}$ of $K$ such that $y \not \equiv 0 \bmod \wp_{i}^{\prime}$. Then, from the above, $e_{K}+c(\eta)=v_{\wp_{i}^{\prime}}\left(b_{0} x^{p-1} y \pi_{K}^{e_{K}+c(\eta)}\right)=v_{\wp_{i}^{\prime}}\left(x_{2}-x^{p}\right)$. This is a multiple of $e_{K}$, so that $c(\eta)$ is also a multiple of $e_{K}$. That is a contradiction. Therefore, $c\left(\eta^{p}\right)=c(\eta)+e_{K}$. Inductively, we obtain $c\left(\eta^{p^{a}}\right)=c(\eta)+a e_{K}$ for all natural numbers $a$.

Furthermore, from (3),

$$
f\left(\eta^{p}\right) \equiv b_{0} \frac{x^{p-1} y}{x^{p}} \equiv b_{0} \frac{y}{x} \equiv b_{0} f(\eta) \bmod p .
$$

This means that $f\left(\eta^{p^{a}}\right)=b_{0}^{a} f(\eta)$ for all natural numbers $a$. The proof is complete.

Lemma 1.5. Let $\eta_{1}, \ldots, \eta_{s}$ be elements in $E_{K} \backslash K_{T}$ such that $c\left(\eta_{1}\right)=$ $\ldots=c\left(\eta_{s}\right)>e_{K} /(p-1)$ and $\left\{f\left(\eta_{1}\right), \ldots, f\left(\eta_{s}\right)\right\}$ is an $\mathbb{F}_{p}$-independent system. Then $\eta_{1}, \ldots, \eta_{s}$ are $\mathbb{Z}$-independent.

Pr o of. Suppose that $\eta_{1}, \ldots, \eta_{s}$ are $\mathbb{Z}$-dependent, that is, $\eta_{1}^{e_{1}} \ldots \eta_{s}^{e_{s}}=1$ with some $e_{1}, \ldots, e_{s} \in \mathbb{Z}$. We may assume $e_{i} \neq 0$ for all $i$.

Let $e_{i}=a_{i} p^{b_{i}}\left(\mathbb{Z} \ni a_{i} \not \equiv 0 \bmod p, 0 \leq b_{i} \in \mathbb{Z}, i=1, \ldots, s\right)$. Then from Lemmas 1.2 and 1.4 we have

$$
c\left(\eta_{i}^{e_{i}}\right)=c\left(\eta_{i}^{p^{b_{i}}}\right)=c\left(\eta_{i}\right)+b_{i} e_{K} \quad \text { and } \quad f\left(\eta_{i}^{e_{i}}\right)=a_{i} f\left(\eta_{i}^{p^{b_{i}}}\right)=b_{0}^{b_{i}} a_{i} f\left(\eta_{i}\right) .
$$

We denote by $\beta$ the minimum of $\left\{b_{1}, \ldots, b_{s}\right\}$ and assume, without loss of generality, $\beta=b_{1}=\ldots=b_{t}<b_{t+1}, \ldots, b_{s}$ with some $t(1 \leq t \leq s)$.

From our assumption,

$$
\sum_{i=1}^{t} f\left(\eta_{i}^{e_{i}}\right)=\sum_{i=1}^{t} b_{0}^{b_{i}} a_{i} f\left(\eta_{i}\right) \not \equiv 0 \bmod p .
$$


Now, $c\left(\eta_{i}^{e_{i}}\right)=c\left(\eta_{i}\right)+b_{i} e_{K}=c\left(\eta_{i}\right)+\beta e_{K}$ for all $i=1, \ldots, t$. So, from Lemma 1.2, $c\left(\prod_{i=1}^{t} \eta_{i}^{e_{i}}\right)=c\left(\eta_{1}\right)+\beta e_{K}<c\left(\eta_{j}^{e_{j}}\right)$ for all $t+1 \leq j \leq s$. Therefore, $c\left(\prod_{i=1}^{s} \eta_{i}^{e_{i}}\right)=c\left(\prod_{i=1}^{t} \eta_{i}^{e_{i}}\right)=c\left(\eta_{1}\right)+\beta e_{K}$. This contradicts $\prod_{i=1}^{s} \eta_{i}^{e_{i}}=1$ (whose level is $\infty$ ) and the lemma is proved.

In the end we investigate the action of $J$ on the Fermat quotient of a real unit.

LEMMA 1.6. For any $\eta \in E_{K^{+}} \backslash K_{T}, f(\eta)^{J}=(-1)^{c(\eta)} f(\eta)$ if $K_{V}$ is imaginary, and $f(\eta)^{J}=f(\eta)$ if $K_{V}$ is real.

Proof. First, we assume that $K_{V}$ is imaginary. Let $\eta \equiv x+y \pi_{K}^{c(\eta)}$ $\bmod \pi_{K}^{c(\eta)+1}$ according to Lemma 1.1. From Lemmas 1.2 and 1.3,

$$
\eta=\eta^{J} \equiv x^{J}+y^{J}(-1)^{c(\eta)} \pi_{K}^{c(\eta)} \bmod \pi_{K}^{c(\eta)+1} .
$$

Therefore,

$$
f(\eta)=f\left(\eta^{J}\right) \equiv \frac{y^{J}(-1)^{c(\eta)}}{x^{J}} \equiv(-1)^{c(\eta)}\left(\frac{y}{x}\right)^{J} \equiv(-1)^{c(\eta)} f(\eta)^{J} \bmod p .
$$

When $K_{V}$ is real, $\eta=\eta^{J} \equiv x^{J}+y^{J} \pi_{K}^{c(\eta)} \bmod \pi_{K}^{c(\eta)+1}$. Thus, $f(\eta)=f\left(\eta^{J}\right) \equiv$ $(y / x)^{J} \equiv f(\eta)^{J}$ as desired.

2. A basis of units modulo units of $K_{T}^{+}$. Let the notation be as before. In this section, we shall prove the existence of a set of representatives of a basis of $E^{m p^{\delta} e_{K}} E_{K_{T}^{+}} / E_{K_{T}^{+}}$which satisfies some conditions on the Fermat quotient and the level.

When $K_{T}$ is imaginary, let

$$
O_{K_{T}} /(p)=\left(O_{K_{T}} /(p)\right)^{+} \oplus\left(O_{K_{T}} /(p)\right)^{-}
$$

be the decomposition associated with $(1+J) / 2$ and $(1-J) / 2$. Then it is easy to see that

(i) $\operatorname{dim}_{\mathbb{F}_{p}}\left(O_{K_{T}} /(p)\right)^{+}=\operatorname{dim}_{\mathbb{F}_{p}}\left(O_{K_{T}} /(p)\right)^{-}=d_{K} / 2$.

(ii) $E_{K}^{e_{K}} \subset \operatorname{Ker}(N) \cdot E_{K_{T}}$ and $E_{K^{+}}^{\left[K^{+}: K_{T}^{+}\right]} \subset \operatorname{Ker}\left(N^{+}\right) \cdot E_{K_{T}^{+}}$, where $N$ and $N^{+}$is the norm map from $K$ to $K_{T}$ and from $K^{+}$to $K_{T}^{+}$, respectively.

The next lemma is due to Washington [6].

Lemma 2.1. Let $E$ be a subgroup of $E_{K}$ of finite index and let $\eta$ be a non-torsion element of $E$. If $v_{\wp_{i}^{\prime}}\left(\log _{p} \eta\right)$ is sufficiently large for all primes $\wp_{i}^{\prime}(i=1, \ldots, g)$ then $\eta$ is a pth power in E. Here, we consider $v_{\wp_{i}^{\prime}}\left(\log _{p} \eta\right)$ and $\log _{p} \eta$ in the localization of $K$ with respect to $\wp_{i}^{\prime}$.

Proof. If $\eta$ is not a $p$ th power in $E$, then we can take $u_{2}, \ldots, u_{r} \in E$ $\left(r=\operatorname{rank}_{\mathbb{Z}} E_{K}\right)$ such that $\left\{\eta, u_{2}, \ldots, u_{r}\right\}$ generates a subgroup $E^{\prime}$ of $E$ of 
finite index prime to $p$. Let $\mathrm{R}_{p}(*)$ be the $p$-adic regulator of $*$ (see Washington [7]). From our assumption, $\mathrm{R}_{p}\left(E^{\prime}\right) \equiv 0 \bmod \wp_{i}^{\prime c}$ for all $\wp_{i}^{\prime} \mid p$, where $c$ is sufficiently large. Now,

$$
\mathrm{R}_{p}\left(E^{\prime}\right)=\left[E_{K}: E\right]\left[E: E^{\prime}\right] \mathrm{R}_{p}\left(E_{K}\right) \neq 0
$$

by our assumption $\left(\mathrm{A}_{3}\right)$. So, $v_{p}\left(\mathrm{R}_{p}\left(E^{\prime}\right)\right)=v_{p}\left(\left[E_{K}: E\right]\right)+v_{p}\left(\mathrm{R}_{p}\left(E_{K}\right)\right)$. The right hand side depends only on $K$ and $E$. But the left hand side is sufficiently large. That is a contradiction and the proof is complete.

Next we prove a relation between $v_{\wp_{i}^{\prime}}\left(\log _{p} \eta\right)$ and the level of $\eta$.

LEMMA 2.2. Let $\eta$ be any element of $E_{K} \backslash K_{T}$ and $\wp_{i}^{\prime}(i=1, \ldots, g)$ the prime of $K$ over $p$. Suppose $N(\eta)=1$ and $c(\eta)>e_{K} /(p-1)$. Then

$$
v_{\wp_{i}^{\prime}}\left(\log _{p} \eta\right) \geq \min \left\{c(\eta)+1-v_{p}\left(e_{K}\right) e_{K}, c(\eta)\right\} \quad \text { for all } \wp_{i}^{\prime} .
$$

Pro of. Let $\eta \equiv x+y \pi_{K}^{c(\eta)} \bmod \pi_{K}^{c(\eta)+1}$ according to Lemma 1.1. Let $c=$ $c(\eta)$. Fix any prime $\wp_{i}^{\prime} \mid p$. From the assumption, $1=N(\eta) \equiv x^{e_{K}} \bmod \pi_{K}^{c}$. Observe that the $\pi_{K}$-order of $x^{e_{K}}-1$ is a multiple of $e_{K}$ and $c$ is not a multiple of $e_{K}$ by Lemma 1.1. Thus $x^{e_{K}} \equiv 1 \bmod \pi_{K}^{c+1}$. As $c>e_{K} /(p-1)$, we have $v_{\wp_{i}^{\prime}}\left(\log _{p} x^{e_{K}}\right) \geq c+1$ (see Lemma 5.5 of Washington [7]). Thus, $v_{\wp_{i}^{\prime}}\left(e_{K}\right)+v_{\wp_{i}^{\prime}}\left(\log _{p} x\right) \geq c+1$. From $v_{\wp_{i}^{\prime}}\left(e_{K}\right)=v_{p}\left(e_{K}\right) e_{K}$, we obtain $v_{\wp_{i}^{\prime}}\left(\log _{p} x\right) \geq c+1-v_{p}\left(e_{K}\right) e_{K}$. There exists $y_{1} \in O_{K}$ such that $\eta=x+y_{1} \pi_{K}^{c}$ and $y_{1} \equiv y \bmod \pi_{K}$. Then, since $\log _{p} \eta=\log _{p} x+\log _{p}\left(1+y_{1} \pi_{K}^{c} / x\right)$, we have

$$
\begin{aligned}
v_{\wp_{i}^{\prime}}\left(\log _{p} \eta\right) & \geq \min \left\{v_{\wp_{i}^{\prime}}\left(\log _{p} x\right), v_{\wp_{i}^{\prime}}\left(\log _{p}\left(1+\frac{y_{1}}{x} \pi_{K}^{c}\right)\right)\right\} \\
& \geq \min \left\{c+1-v_{p}\left(e_{K}\right) e_{K}, c\right\} .
\end{aligned}
$$

The lemma is proved.

For any natural number $c$, we define

$$
F_{K}^{c}=\left\{f(\eta): \eta \in E_{K^{+}} \backslash K_{T} \text { such that } c(\eta)=c\right\} \subset O_{K_{T}} /(p) .
$$

Lemma 2.3. (I) If $K_{T}$ and $K_{V}$ are imaginary, then $F_{K}^{c} \subset\left(O_{K_{T}} /(p)\right)^{+}$ if $c$ is even, and $F_{K}^{c} \subset\left(O_{K_{T}} /(p)\right)^{-}$if $c$ is odd. Moreover, $\operatorname{dim}_{\mathbb{F}_{p}} F_{K}^{c} \leq \frac{1}{2} d_{K}$.

(II) If $K_{T}$ is imaginary and $K_{V}$ is real, then $F_{K}^{c} \subset\left(O_{K_{T}} /(p)\right)^{+}$for all $c$, and $\operatorname{dim}_{\mathbb{F}_{p}} F_{K}^{c} \leq \frac{1}{2} d_{K}$.

(III) If $K_{T}$ is real and $K_{V}$ is imaginary, then $e_{K}$ is even and $c(\eta)$ is even for all $\eta \in E_{K^{+}} \backslash K_{T}$. Obviously, $F_{K}^{c} \subset\left(O_{K_{T}} /(p)\right)=\left(O_{K_{T}} /(p)\right)^{+}$and $\operatorname{dim}_{\mathbb{F}_{p}} F_{K}^{c} \leq d_{K}$.

(IV) If $K_{T}$ and $K_{V}$ are real, then $F_{K}^{c} \subset\left(O_{K_{T}} /(p)\right)=\left(O_{K_{T}} /(p)\right)^{+}$and $\operatorname{dim}_{\mathbb{F}_{p}} F_{K}^{c} \leq d_{K}$.

Proof. (I) Clearly, $K$ is imaginary. Let $\eta \in E_{K^{+}} \backslash K_{T}$ and $\eta \equiv x+$ $y \pi_{K}^{c(\eta)} \bmod \pi_{K}^{c(\eta)+1}$ according to Lemma 1.1. Let $c=c(\eta)$. Since $K_{V}$ is imaginary, the statement follows from Lemma 1.6 and (i). 
(II) In this case, our statement follows easily from Lemma 1.6 and (i).

(III) Let $\eta \in E_{K^{+}} \backslash K_{T}$ and $\eta \equiv x+y \pi_{K}^{c(\eta)} \bmod \pi_{K}^{c(\eta)+1}$ according to Lemma 1.1. Let $c=c(\eta)$. Because the order of $J$ is $2, e_{K}$ is clearly even. From Lemma 1.3,

$$
\eta=\eta^{J} \equiv x^{J}+y^{J}(-1)^{c} \pi_{K}^{c} \bmod \pi_{K}^{c+1} .
$$

Here, $x^{J}=x$ and $y^{J}=y$ because $K_{T}$ is real. So, $x+y \pi_{K}^{c} \equiv x+y(-1)^{c} \pi_{K}^{c}$ $\bmod \pi_{K}^{c+1}$. This means that $c$ is even.

(IV) It is clear.

REMARK 3. We have $r=\operatorname{rank}_{\mathbb{Z}}\left(E_{K^{+}} / E_{K_{T}^{+}}\right)=\operatorname{rank}_{\mathbb{Z}} E_{K^{+}}-\operatorname{rank}_{\mathbb{Z}} E_{K_{T}^{+}}$. Hence we easily observe that:

$r=\frac{1}{2} d_{K}\left(e_{K}-1\right)$ and $\operatorname{dim}_{\mathbb{F}_{p}} F_{K}^{c} \leq \frac{1}{2} d_{K}$ in the case (I) or (II).

$r=d_{K}\left(\frac{1}{2} e_{K}-1\right)$ and $\operatorname{dim}_{\mathbb{F}_{p}} F_{K}^{c} \leq d_{K}$ in the case (III).

$r=d_{K}\left(e_{K}-1\right)$ and $\operatorname{dim}_{\mathbb{F}_{p}} F_{K}^{c} \leq d_{K}$ in the case (IV).

TheOrem 2.4. Let $E \supset E_{K_{T}^{+}}$be a subgroup of $E_{K^{+}}$of finite index. Let $r=\operatorname{rank}_{\mathbb{Z}}\left(E_{K^{+}} / E_{K_{T}^{+}}\right)$. Then there exists a set of representatives $\left\{\eta_{1}, \ldots, \eta_{r}\right\}$ of a basis of $E^{m p^{\delta} e_{K}} E_{K_{T}^{+}} / E_{K_{T}^{+}}$such that

(1) $c\left(\eta_{i}\right)>e_{K} /(p-1)(i=1, \ldots, r)$.

(2) $N^{+}\left(\eta_{i}\right)=1(i=1, \ldots, r)$.

(3) $c_{1} \leq c_{2} \leq \ldots \leq c_{r}$ where $c_{i}=c\left(\eta_{i}\right)$.

(4) Let $S_{j}=\left\{\eta_{i}: c\left(\eta_{i}\right) \equiv j \bmod e_{K}\right\} \quad\left(1 \leq j<e_{K}\right.$ and $j$ is even only if $K_{T}$ is real and $K_{V}$ is imaginary). Then $\sharp S_{j}=\frac{1}{2} d_{K}$ (d $d_{K}$ resp.) when $K_{T}$ is imaginary (resp. real) and $\left\{f\left(\eta_{i}\right): \eta_{i} \in S_{j}\right\}$ is an $\mathbb{F}_{p}$-independent system for each $j$ which defines $S_{j}$.

Proof. Let $\left\{\xi_{1}, \ldots, \xi_{r}\right\}, \xi_{i} \in E$, be a set of representatives of a basis of $E^{m} E_{K_{T}^{+}} / E_{K_{T}^{+}}$. Observe that $E^{m} E_{K_{T}^{+}} / E_{K_{T}^{+}}$is torsion-free. From Lemma 1.4, $c\left(\xi_{i}^{p^{\delta}}\right)>e_{K} /(p-1)$. From (ii), as $\left[K^{+}: K_{T}^{+}\right]=e_{K}$ or $e_{K} / 2$, we have $\xi_{i}^{p^{\delta} e_{K}} \in \operatorname{Ker}\left(N^{+}\right) \cdot E_{K_{T}^{+}}$. Therefore,

$$
\xi_{i}^{p^{\delta} e_{K}}=\eta_{i} u_{i} \quad \text { with some } \eta_{i} \in \operatorname{Ker}\left(N^{+}\right) \cap E \text { and } u_{i} \in E_{K_{T}^{+}}, 1 \leq i \leq r .
$$

Here, $\left\{\eta_{1}, \ldots, \eta_{r}\right\}$ is also a set of representatives of a basis of the quotient $E^{m p^{\delta} e_{K}} E_{K_{T}^{+}} / E_{K_{T}^{+}}$that satisfies (1) and (2). And (3) is satisfied by an appropriate change of indices.

Now we define the condition $\left(\mathrm{C}_{s}\right)$ for $1 \leq s<r:\left\{f\left(\eta_{i}\right): c\left(\eta_{i}\right) \equiv\right.$ $\left.j \bmod e_{K}, 1 \leq i \leq s\right\}$ is an $\mathbb{F}_{p}$-independent system for all $j\left(1 \leq j<e_{K}\right.$, $j$ is even if $K_{T}$ is real and $K_{V}$ is imaginary). Clearly, $\left(\mathrm{C}_{1}\right)$ is true. Suppose that $\left(\mathrm{C}_{s}\right)$ is valid. Let $c\left(\eta_{s+1}\right)=l$. If $\left\{f\left(\eta_{i}\right): c\left(\eta_{i}\right) \equiv l \bmod e_{K}, 1 \leq i \leq s\right\}$ 
$\cup\left\{f\left(\eta_{s+1}\right)\right\}$ is an $\mathbb{F}_{p}$-independent system, then $\left(\mathrm{C}_{s+1}\right)$ is valid. If it is not $\mathbb{F}_{p}$-independent, then

$$
f\left(\eta_{s+1}\right)=\sum_{1 \leq i \leq s, c_{i} \equiv l \bmod e_{K}} a_{i} b_{0}^{\alpha_{i}} f\left(\eta_{i}\right) \quad \text { with some } a_{i} \in \mathbb{Z},
$$

where $\alpha_{i}=\frac{1}{e_{K}}\left(c\left(\eta_{s+1}\right)-c\left(\eta_{i}\right)\right)$. We have $c\left(\eta_{i}^{a_{i} p^{\alpha_{i}}}\right)=c\left(\eta_{s+1}\right)$ and $f\left(\eta_{i}^{a_{i} p^{\alpha_{i}}}\right)=$ $a_{i} b_{0}^{\alpha_{i}} f\left(\eta_{i}\right)$. Then

$$
f\left(\prod_{1 \leq i \leq s, c_{i} \equiv l \bmod e_{K}} \eta_{i}^{a_{i} p^{\alpha_{i}}}\right)=\sum_{1 \leq i \leq s, c_{i} \equiv l \bmod e_{K}} a_{i} b_{0}^{\alpha_{i}} f\left(\eta_{i}\right)=f\left(\eta_{s+1}\right) .
$$

So, letting

$$
\eta_{s+1}^{\prime}=\eta_{s+1}\left(\prod_{1 \leq i \leq s, c_{i} \equiv l \bmod e_{K}} \eta_{i}^{a_{i} p^{\alpha_{i}}}\right)^{-1},
$$

we get $c\left(\eta_{s+1}^{\prime}\right)>c\left(\eta_{s+1}\right)$. Now $\left\{\eta_{1}, \ldots, \eta_{s}, \eta_{s+1}^{\prime}, \eta_{s+2}, \ldots, \eta_{r}\right\}$ is also a set of representatives that satisfies (1) and (2). By means of some permutation of $\left\{\eta_{s+1}^{\prime}, \eta_{s+2}, \ldots, \eta_{r}\right\}$, we may write it $\left\{\eta_{s+1}, \eta_{s+2}, \ldots, \eta_{r}\right\}$ again with $c\left(\eta_{s+1}\right) \leq \ldots \leq c\left(\eta_{r}\right)$. Then, further, we repeat the above procedure for $\eta_{s+1}$. Lemmas 2.1 and 2.2 imply that the procedure must stop after a finite number of steps. Hence $\left(\mathrm{C}_{s+1}\right)$ becomes true. So, inductively, we get $\left\{\eta_{1}, \ldots, \eta_{r}\right\}$ as desired.

Note that, in this theorem, the sum of $\sharp S_{j}$ for $1 \leq j<e_{K}$ ( $j$ is even when $K_{T}$ is real and $K_{V}$ is imaginary) is equal to $r$ by Remark 3 .

3. A formula for index of subgroups. Let $E$ and $E^{\prime}$ be subgroups of $E_{K^{+}}$such that $E \supset E^{\prime} \supset E_{K_{T}^{+}}$and $\left[E_{K^{+}}: E^{\prime}\right]<\infty$. Let $\left\{\eta_{i}\right\}$ and $\left\{\theta_{i}\right\}$ be as in Theorem 2.4 for $E$ and $E^{\prime}$, respectively.

For $\eta \in E_{K}$, let $\bar{\eta}$ denote $\eta \bmod E_{K_{T}^{+}}$, and $c(\bar{\eta})=c(\eta)$ and $f(\bar{\eta})=f(\eta)$. They are well defined because $c(\eta u)=c(\eta)$ and $f(\eta u)=f(\eta)$ for any $u \in E_{K_{T}^{+}}$.

We define $d_{0}$ to be $\frac{1}{2} d_{K}$ if $K_{T}$ is imaginary (in the case (I) or (II) in Lemma 2.3) and $d_{K}$ if $K_{T}$ is real (in the case (III) or (IV) in Lemma 2.3). We let $R=\{1, \ldots, r\}$ and $B_{l}=\left\{(l-1) d_{0}+1, \ldots, l d_{0}\right\}\left(1 \leq l<e_{K}\right)$, in the case (I), (II) or (IV) in Lemma 2.3. Moreover we define $B_{l}$ in the case (III) as follows:

$$
B_{l}=\left\{\left(\frac{l}{2}-1\right) d_{0}+1, \ldots, \frac{l}{2} d_{0}\right\} \quad \text { for } 1<l<e_{K} \text { and } l \text { even. }
$$

Then $R$ is the union of all $B_{l}$.

We permute $\bar{\eta}_{1}, \ldots, \bar{\eta}_{r}$ and $\bar{\theta}_{1}, \ldots, \bar{\theta}_{r}$ such that $c\left(\eta_{i}\right) \equiv l \bmod e_{K}$ for all $i \in B_{l}$ and $c\left(\theta_{j}\right) \equiv l \bmod e_{K}$ for all $j \in B_{l}$. 
We let, for all $j=1, \ldots, r$,

(5) $\quad \bar{\theta}_{j}=\prod_{i=1}^{r} \bar{\eta}_{i}^{a_{j i} p^{e_{j i}}} \quad$ where $a_{j i} \in \mathbb{Z}$ is 0 or prime to $p$, and $0 \leq e_{j i} \in \mathbb{Z}$.

Then

$$
\begin{aligned}
\operatorname{det}\left(a_{j i} p^{e_{j i}}\right) & =\left[E^{m p^{\delta} e_{K}} E_{K_{T}^{+}} / E_{K_{T}^{+}}:\left(E^{\prime}\right)^{m p^{\delta} e_{K}} E_{K_{T}^{+}} / E_{K_{T}^{+}}\right] \\
& =\left[E^{m} E_{K_{T}^{+}} / E_{K_{T}^{+}}:\left(E^{\prime}\right)^{m} E_{K_{T}^{+}} / E_{K_{T}^{+}}\right] \\
& =\left[E: E^{\prime}\right] \times(\text { a natural number prime to } p)
\end{aligned}
$$

since $E^{m} E_{K_{T}^{+}} / E_{K_{T}^{+}}$and $\left(E^{\prime}\right)^{m} E_{K_{T}^{+}} / E_{K_{T}^{+}}$are torsion-free and $m$ is prime to $p$ by our assumption $\left(\mathrm{A}_{2}\right)$. Consequently, we have:

LEMma 3.1. Let $E$ and $E^{\prime}$ be subgroups of $E_{K^{+}}$such that $E \supset E^{\prime} \supset$ $E_{K_{T}^{+}}$and $\left[E_{K^{+}}: E^{\prime}\right]<\infty$. Then $v_{p}\left(\operatorname{det}\left(a_{j i} p^{e_{j i}}\right)\right)=v_{p}\left(\left[E: E^{\prime}\right]\right)$.

Next we prove a formula for index $\left[E: E^{\prime}\right]$.

TheOREM 3.2. Let $E$ and $E^{\prime}$ be subgroups of $E_{K^{+}}$such that $E \supset E^{\prime} \supset$ $E_{K_{T}^{+}}$and $\left[E_{K^{+}}: E^{\prime}\right]<\infty$. Let $\left\{\eta_{j}\right\}$ and $\left\{\theta_{j}\right\}$ be as in Theorem 2.4 for $E$ and $E^{\prime}$, respectively. Then

$$
v_{p}\left(\left[E: E^{\prime}\right]\right)=\frac{1}{e_{K}}\left\{\sum_{j=1}^{r} c\left(\bar{\theta}_{j}\right)-\sum_{j=1}^{r} c\left(\bar{\eta}_{j}\right)\right\} .
$$

Proof. By the properties of level given in Section 1,

$$
c\left(\bar{\theta}_{j}\right)=\min \left\{c\left(\bar{\eta}_{i}{ }^{a_{j i} p^{e_{j i}}}\right): 1 \leq i \leq r\right\} .
$$

Define $A_{j}=\left\{i \in R: c\left(\bar{\theta}_{j}\right)=c\left(\bar{\eta}_{i}^{a_{j i} p^{e_{j i}}}\right)\right\}, 1 \leq j \leq r$. Clearly, $A_{j}$ is non-empty and $A_{j} \subset B_{l}$ if $j \in B_{l}$. Further,

$$
f\left(\bar{\theta}_{j}\right)=\sum_{i \in A_{j}} f\left(\bar{\eta}_{i}^{a_{j i} p^{e_{j i}}}\right)=\sum_{i \in A_{j}} a_{j i} b_{0}^{e_{j i}} f\left(\bar{\eta}_{i}\right) \quad \text { for all } j \in R .
$$

Since $\left\{f\left(\bar{\eta}_{i}\right)\right\}_{i \in B_{l}}$ and $\left\{f\left(\bar{\theta}_{j}\right)\right\}_{j \in B_{l}}$ are $\mathbb{F}_{p}$-independent systems, it follovs that

$$
\operatorname{det}\left(a_{j i}^{\prime} b_{0}^{e_{j i}}\right)_{j, i \in B_{l}} \not \equiv 0 \bmod p \quad \text { for all } l,
$$

where $a_{j i}^{\prime}=a_{j i}$ if $i \in A_{j}$ and $a_{j i}^{\prime}=0$ if $i \notin A_{j}$.

Now we define $P_{l}\left(1 \leq l<e_{K}, l\right.$ is even in the case (III) in Lemma 2.3) to be the set of all permutations on $B_{l}$ and $P_{l}^{\prime}=\left\{\tau_{l} \in P_{l}: \tau_{l}(j) \in A_{j}\right.$ for all $\left.j \in B_{l}\right\}$.

Then, for each $l$,

$$
\operatorname{det}\left(a_{j i}^{\prime} b_{0}^{e_{j i}}\right)_{j, i \in B_{l}}=\sum_{\tau_{l} \in P_{l}^{\prime}}\left(\operatorname{sgn}\left(\tau_{l}\right) \cdot \prod_{j \in B_{l}} a_{j \tau_{l}(j)} b_{0}^{e_{j \tau_{l}(j)}}\right) .
$$


From (6) and (7), we see that $P_{l}^{\prime}$ is non-empty for every $l$. Let $P$ be the set of all permutations on $R$ and $P^{\prime}=\left\{\varrho \in P: \varrho(j) \in A_{j}\right.$ for all $\left.j \in R\right\}$. It is clear that any element of $P^{\prime}$ is a product of $\tau_{l} \in P_{l}^{\prime}\left(1 \leq l<e_{K}, l\right.$ is even in the case (III) of Lemma 2.3) and the restriction of each $\varrho \in P^{\prime}$ to $B_{l}$ is an element of $P_{l}^{\prime}$. So, we see that $P^{\prime}$ is non-empty. In general, for any $\varrho \in P, c\left(\bar{\theta}_{j}\right) \leq c\left(\bar{\eta}_{\varrho(j)}^{a_{j \varrho(j)} p^{e_{j \varrho(j)}}}\right)$ for all $j \in R$, while for each $\varrho \in P \backslash P^{\prime}$, $c\left(\bar{\theta}_{j}\right)<c\left(\bar{\eta}_{\varrho(j)}^{a_{j \varrho(j)} p^{e_{j \varrho(j)}}}\right)$ with some $j \in R$. Therefore, for each $\varrho \in P \backslash P^{\prime}$,

$$
\sum_{j=1}^{r} c\left(\bar{\theta}_{j}\right)<\sum_{j=1}^{r} c\left(\bar{\eta}_{\varrho(j)}^{a_{j \varrho(j)} p^{e_{j \varrho(j)}}}\right) .
$$

For $\varrho \in P^{\prime}$, by the definition,

$$
\sum_{j=1}^{r} c\left(\bar{\theta}_{j}\right)=\sum_{j=1}^{r} c\left(\bar{\eta}_{\varrho(j)}^{a_{j \varrho(j)} p^{e_{j \varrho(j)}}}\right) .
$$

Since

$\sum_{j=1}^{r} c\left(\bar{\eta}_{\varrho(j)}^{a_{j \varrho(j)} p^{e_{j \varrho(j)}}}\right)=\sum_{j=1}^{r}\left\{c\left(\bar{\eta}_{\varrho(j)}\right)+e_{K} e_{j \varrho(j)}\right\}=\sum_{j=1}^{r} c\left(\bar{\eta}_{\varrho(j)}\right)+e_{K} \sum_{j=1}^{r} e_{j \varrho(j)}$,

we have

$$
\sum_{j=1}^{r} e_{j \varrho(j)}=\frac{1}{e_{K}}\left\{\sum_{j=1}^{r} c\left(\bar{\theta}_{j}\right)-\sum_{j=1}^{r} c\left(\bar{\eta}_{j}\right)\right\} \quad \text { for all } \varrho \in P^{\prime} .
$$

Similarly, from (8),

$$
\sum_{j=1}^{r} e_{j \varrho(j)}>\frac{1}{e_{K}}\left\{\sum_{j=1}^{r} c\left(\bar{\theta}_{j}\right)-\sum_{j=1}^{r} c\left(\bar{\eta}_{j}\right)\right\} \quad \text { for all } \varrho \in P \backslash P^{\prime} .
$$

From (7) and (9),

$$
\begin{aligned}
\prod_{l} \operatorname{det}\left(a_{j i}^{\prime} b_{0}^{e_{j i}}\right)_{j, i \in B_{l}} \\
\quad=\sum_{\varrho \in P^{\prime}}\left(\operatorname{sgn}(\varrho) \cdot \prod_{j=1}^{r} a_{j \varrho(j)} b_{0}^{e_{j \varrho(j)}}\right) \\
=\sum_{\varrho \in P^{\prime}}\left(\operatorname{sgn}(\varrho) \cdot b_{0}^{\sum_{j=1}^{r} e_{j \varrho(j)}} \cdot \prod_{j=1}^{r} a_{j \varrho(j)}\right) \\
=b_{0}^{\left(1 / e_{K}\right)\left(\sum_{j=1}^{r} c\left(\bar{\theta}_{j}\right)-\sum_{j=1}^{r} c\left(\bar{\eta}_{j}\right)\right)} \cdot \sum_{\varrho \in P^{\prime}}\left(\operatorname{sgn}(\varrho) \cdot \prod_{j=1}^{r} a_{j \varrho(j)}\right) .
\end{aligned}
$$


Therefore, from (6),

$$
\sum_{\varrho \in P^{\prime}}\left(\operatorname{sgn}(\varrho) \cdot \prod_{j=1}^{r} a_{j \varrho(j)}\right) \not \equiv 0 \bmod p .
$$

Now, we have

$$
\begin{aligned}
& \operatorname{det}\left(a_{j i} p^{e_{j i}}\right)_{1 \leq j, i \leq r} \\
= & \sum_{\varrho \in P^{\prime}}\left(\operatorname{sgn}(\varrho) \cdot \prod_{j=1}^{r} a_{j \varrho(j)} p^{e_{j \varrho(j)}}\right)+\sum_{\varrho \in P \backslash P^{\prime}}\left(\operatorname{sgn}(\varrho) \cdot \prod_{j=1}^{r} a_{j \varrho(j)} p^{e_{j \varrho(j)}}\right) \\
= & \sum_{\varrho \in P^{\prime}}\left(\operatorname{sgn}(\varrho) \cdot p^{\sum_{j=1}^{r} e_{j \varrho(j)}} \cdot \prod_{j=1}^{r} a_{j \varrho(j)}\right) \\
& +\sum_{\varrho \in P \backslash P^{\prime}}\left(\operatorname{sgn}(\varrho) \cdot p^{\sum_{j=1}^{r} e_{j \varrho(j)}} \cdot \prod_{j=1}^{r} a_{j \varrho(j)}\right) \\
= & p^{\left(1 / e_{K}\right)\left(\sum_{j=1}^{r} c\left(\bar{\theta}_{j}\right)-\sum_{j=1}^{r} c\left(\bar{\eta}_{j}\right)\right)} \cdot \sum_{\varrho \in P^{\prime}}\left(\operatorname{sgn}(\varrho) \cdot \prod_{j=1}^{r} a_{j \varrho(j)}\right) \\
& +\sum_{\varrho \in P \backslash P^{\prime}}\left(\operatorname{sgn}(\varrho) \cdot p^{\sum_{j=1}^{r} e_{j \varrho(j)}} \cdot \prod_{j=1}^{r} a_{j \varrho(j)}\right) .
\end{aligned}
$$

Combining (10)-(12), we get

$$
v_{p}\left(\operatorname{det}\left(a_{j i} p^{e_{j i}}\right)_{1 \leq j, i \leq r}\right)=\frac{1}{e_{K}}\left\{\sum_{j=1}^{r} c\left(\bar{\theta}_{j}\right)-\sum_{j=1}^{r} c\left(\bar{\eta}_{j}\right)\right\} .
$$

The proof is completed by using Lemma 3.1.

Lemma 3.3. Let $E$ and $E^{\prime}$ be subgroups of $E_{K^{+}}$such that $E \supset E^{\prime} \supset E_{K_{T}^{+}}$ and $\left[E_{K^{+}}: E^{\prime}\right]<\infty$. Let $\left\{\eta_{i}\right\}$ and $\left\{\theta_{i}\right\}$ be as in Theorem 2.4 for $E$ and $E^{\prime}$, respectively. Then $c\left(\bar{\theta}_{i}\right) \geq c\left(\bar{\eta}_{i}\right)$ for all $i=1, \ldots, r$.

P r o o f. Suppose that there exists $t$ such that $1 \leq t \leq r$ and $c\left(\bar{\theta}_{t}\right)<c\left(\bar{\eta}_{t}\right)$. For each $l\left(1 \leq l \leq e_{K}-1, l\right.$ is even in the case (III)), we define

$$
T_{l}=\left\{\bar{\theta}_{h}: 1 \leq h \leq t, c\left(\bar{\theta}_{h}\right) \equiv l \bmod e_{K}\right\} .
$$

Then $\left\{\bar{\theta}_{1}, \ldots, \bar{\theta}_{t}\right\}=\bigcup_{l} T_{l}$ (disjoint union) and $t=\sum_{l} \sharp T_{l}$. Fix any $l$. Then each $f\left(\bar{\theta}_{h}\right)\left(\bar{\theta}_{h} \in T_{l}\right)$ is a linear combination of $f\left(\bar{\eta}_{1}\right), \ldots, f\left(\bar{\eta}_{t-1}\right)$ because $c\left(\bar{\theta}_{1}\right) \leq \ldots \leq c\left(\bar{\theta}_{t}\right)<c\left(\bar{\eta}_{t}\right) \leq \ldots \leq c\left(\bar{\eta}_{r}\right)$. For each $l\left(1 \leq l \leq e_{K}-1, l\right.$ is even in the case (III)), we define

$$
T_{l}^{\prime}=\left\{\bar{\eta}_{i}: 1 \leq i \leq t-1, c\left(\bar{\eta}_{i}\right) \equiv l \bmod e_{K}\right\} .
$$


Then $\left\{\bar{\eta}_{1}, \ldots, \bar{\eta}_{t-1}\right\}=\bigcup_{l} T_{l}^{\prime} \quad$ (disjoint union) and $t-1=\sum_{l} \sharp T_{l}^{\prime}$. Now $f\left(\bar{\theta}_{h}\right)\left(\bar{\theta}_{h} \in T_{l}\right)$ is a linear combination of $\left\{f\left(\bar{\eta}_{i}\right): \bar{\eta}_{i} \in T_{l}^{\prime}\right\}$. Therefore, from Theorem 2.4(4), we have $\sharp T_{l} \leq \sharp T_{l}^{\prime}$ for any $l$, and hence $\sum_{l} \sharp T_{l} \leq \sum_{l} \sharp T_{l}^{\prime}$. This is a contradiction.

Note that, in Theorem 3.2, it does not necessarily hold that

$$
c\left(\bar{\theta}_{i}\right) \equiv c\left(\bar{\eta}_{i}\right) \bmod e_{K} \quad \text { for all } i=1, \ldots, r,
$$

because $c\left(\bar{\theta}_{1}\right)$ is not necessarily congruent to $c\left(\bar{\eta}_{1}\right)$ modulo $e_{K}$.

Observe that we can define integers $l\left(\eta_{i}\right)$ and $u\left(\eta_{i}\right)$ as follows:

$$
c\left(\eta_{i}\right)=l\left(\eta_{i}\right)+u\left(\eta_{i}\right) e_{K}, \quad 1 \leq l\left(\eta_{i}\right)<e_{K}, 0 \leq u\left(\eta_{i}\right) \in \mathbb{Z}, 1 \leq i \leq r .
$$

Let $l\left(\theta_{i}\right)$ and $u\left(\theta_{i}\right)$ be defined in the same way for $\theta_{i}$. From Lemma 3.3,

$$
c\left(\theta_{i}\right)-c\left(\eta_{i}\right)=l\left(\theta_{i}\right)-l\left(\eta_{i}\right)+e_{K}\left\{u\left(\theta_{i}\right)-u\left(\eta_{i}\right)\right\} \geq 0 .
$$

So that, $u\left(\theta_{i}\right) \geq u\left(\eta_{i}\right)$ for all $i=1, \ldots, r$. In addition,

$$
\begin{aligned}
\sum_{i=1}^{r} c\left(\theta_{i}\right)-\sum_{i=1}^{r} c\left(\eta_{i}\right) & =\sum_{i=1}^{r}\left\{l\left(\theta_{i}\right)-l\left(\eta_{i}\right)\right\}+e_{K} \sum_{i=1}^{r}\left\{u\left(\theta_{i}\right)-u\left(\eta_{i}\right)\right\} \\
& =e_{K} \sum_{i=1}^{r}\left\{u\left(\theta_{i}\right)-u\left(\eta_{i}\right)\right\},
\end{aligned}
$$

because $\sum_{i=1}^{r} l\left(\theta_{i}\right)=\sum_{i=1}^{r} l\left(\eta_{i}\right)$ from Theorem 2.4(4).

On the other hand, by Lemma 3.3, we can easily see that the sequence of rational integers $\left\{c\left(\eta_{1}\right), \ldots, c\left(\eta_{r}\right)\right\}$, hence $\left\{u\left(\eta_{1}\right), \ldots, u\left(\eta_{r}\right)\right\}$, depends only on $E$. So we may write $u\left(\eta_{i}\right)=u_{i}(E)$ and $u\left(\theta_{i}\right)=u_{i}\left(E^{\prime}\right)$. Consequently, by means of Theorem 3.2, we have proved the following:

TheOrem 3.4. Let $E$ and $E^{\prime}$ be subgroups of $E_{K^{+}}$such that $E \supset E^{\prime} \supset$ $E_{K_{T}^{+}}$and $\left[E_{K^{+}}: E^{\prime}\right]<\infty$. Let $u_{i}(E)$ and $u_{i}\left(E^{\prime}\right)$ be as above. Then

$$
v_{p}\left(\left[E: E^{\prime}\right]\right)=\sum_{i=1}^{r}\left\{u_{i}\left(E^{\prime}\right)-u_{i}(E)\right\} .
$$

This is a generalization of Theorems B and D. Now, following Dénes, we may call $u_{i}(E)$ the $p$-character of $E$.

\section{References}

[1] A. Brumer, On the units of algebraic number fields, Mathematika 14 (1967), 121-124.

[2] P. Dénes, Über irreguläre Kreiskörper, Publ. Math. Debrecen 3 (1953), 17-23.

[3] -, Über Grundeinheitssysteme der irregulären Kreiskörper von besonderen Kongruenzeigenschaften, ibid. 3 (1954), 195-204. 
[4] P. Dénes, Über den zweiten Faktor der Klassenzahl und den Irregularitätsgrad der irregulären Kreiskörper, ibid. 4 (1956), 163-170.

[5] F. Kurihara, On the p-adic expansion of units of cyclotomic fields, J. Number Theory 32 (1989), 226-253.

[6] L. C. Washington, Units of irregular cyclotomic fields, Illinois J. Math. 23 (1979), 635-647.

[7] -, Introduction to Cyclotomic Fields, Springer, New York, 1997.

Kanagawa Prefectural Yamato-Higashi High-School

1760 Fukami, Yamato, Kanagawa, 242, Japan

E-mail: tshimada@qa2.so-net.or.jp

Received on 15.7.1997

and in revised form on 3.3.1998 